\title{
On the Local Minima of the Order of Appearance Function
}

\author{
Florian Luca and Thato Mosima \\ School of Mathematics, University of the Witwatersrand, Private Bag X3, Wits, Johannesburg 2050, South Africa \\ Correspondence should be addressed to Florian Luca; florian.luca@wits.ac.za
}

Received 17 August 2015; Accepted 20 September 2015

Academic Editor: Pentti Haukkanen

Copyright (C) 2015 F. Luca and T. Mosima. This is an open access article distributed under the Creative Commons Attribution License, which permits unrestricted use, distribution, and reproduction in any medium, provided the original work is properly cited.

The order of appearance $z(n)$ of the positive integer $n$ is the smallest positive integer $k$ such that $n$ divides $F_{k}$, the $k$ th member of the Fibonacci sequence. In this paper, we improve upon some results from (Marques, 2011) concerning local minima of $z(n)$.

\section{Introduction}

Let $\left\{F_{m}\right\}_{m \geq 0}$ be the Fibonacci sequence given by $F_{0}=0, F_{1}=$ 1 , and $F_{n+2}=F_{n+1}+F_{n}$ for all $n \geq 0$. For a positive integer $n$, let $z(n)$ be the order of appearance of $n$ in the Fibonacci sequence, which is the minimal positive integer $k$ such that $n \mid F_{k}$. It is known that $z(n)$ always exists and in fact $z(n) \leq \sigma(n)$, where $\sigma(n)$ is the sum of divisors of $n$. Let us say that $N$ is a local minimum for the function $z(n)$ if $z(N)<\min \{z(N-1), z(N+$ $1)\}$. It is not hard to prove that if $N=F_{n}$ for some positive integer $n \neq 3$ (so $N \neq 2$ ), then $N$ is a local minimum for $z(n)$ (see Page 1 in [1]).

In Theorem 1.1 in [1], Marques exhibited a family of positive integers which are not members of the Fibonacci sequence but are local minima for $z(n)$. That family is

$$
\mathscr{M}_{1}=\left\{\frac{F_{m k}}{F_{k}}: k \geq 3, m \geq m_{k}\right\},
$$

where $m_{k} \geq 5$ is some fixed number depending on $k$ which is not computable from the arguments in [1]. This problem was revisited in [2], where a different family of local minima is given; namely,

$$
\mathscr{M}_{2}=\left\{\frac{F_{m k}+\varepsilon F_{k}}{F_{k}-\varepsilon}: \varepsilon \in\{ \pm 1\}, 12|k| m, m>m_{\varepsilon, k}\right\},
$$

where as before $m_{\varepsilon, k}$ depends on $\varepsilon$ and $k$ and is not computable from the arguments in [2].

None of the above two families gives us too many examples. Indeed, let be $x$ a large positive real number and put $\mathscr{A}(x)=\mathscr{A} \cap[1, x]$. Assume that $N \in \mathscr{M}_{1}(x) \cup \mathscr{M}_{2}(x)$. Then, using the Binet formula

$$
F_{i}=\frac{\alpha^{i}-\beta^{i}}{\alpha-\beta}, \quad \text { where }(\alpha, \beta)=\left(\frac{1+\sqrt{5}}{2}, \frac{1-\sqrt{5}}{2}\right)
$$

valid for all integers $i \geq 0$, it follows that $N$ is determined in at most 3 ways by a pair of parameters $(m, k)$ with $k \geq 3$ such that

$$
m(k-1) \leq(c+o(1)) \log x \quad \text { as } x \longrightarrow \infty,
$$

where $c=(\log \alpha)^{-1}$. Using the classical estimates on the summatory function of the number of divisors function we get that

$$
\#\left(\mathscr{M}_{1}(x) \cup \mathscr{M}_{2}(x)\right)=O(\log x \log \log x) .
$$

Before we formulate the main result of this paper we need one more notion. A prime factor $p$ of $F_{m}$ is called primitive if $z(p)=m$. A celebrated result of Carmichael [3] (see [4] for the most general result of this type) asserts that $p$ always exists whenever $m \geq 13$. The main result of this paper is the following.

Theorem 1. Let $n \geq 15$ and

$$
N=\frac{F_{n}}{a},
$$

where $a$ is a divisor of $F_{n}$ subject to the following restrictions:

(i) $a \leq F_{n}^{1 / 5}$;

(ii) there exists a primitive prime factor $p$ of $F_{n}$ such that $p+a$. 
Then $n$ is a local minimum for $z(n)$. Furthermore, each such $N$ is representable in a unique way as $N=F_{n} /$ a for some integers $n \geq 15$ and a satisfying (i) and (ii) above, and $N$ is not a Fibonacci number whenever $a \geq 2$.

The inequality $F_{a+b} \geq F_{a} F_{b}$ is valid for all positive integers $a$ and $b$. To prove it, fix $a$, note that it trivially holds for $b \in\{1,2\}$, and then use induction on $b$ and the recurrence formula for the Fibonacci numbers to show that it holds for all $b \geq 1$. In particular, $F_{k} \leq F_{m k}^{1 / m}$. Thus, if $N \in \mathscr{M}_{1}$, then

$$
N=\frac{F_{m k}}{F_{k}},
$$

where if we put $n:=m k$ and $a:=F_{k}$, then $a \leq F_{m k}^{1 / m} \leq F_{n}^{1 / 5}$ because $m \geq 5$. Additionally, $n \geq 15$ because $k \geq 3$ and $m \geq$ 5. Further, the number $N$ is clearly divisible by a primitive divisor of $F_{n}$ (in fact, by any of the primitive divisors of $F_{n}$ ). This argument shows that the set $\mathscr{M}_{1}$ is contained in the set of numbers $N$ satisfying the conditions of Theorem 1 . Now Theorem 1 says that in fact the parameter $m_{k}$ from $\mathscr{M}_{1}$ can always be taken to be 5 . Putting $\mathscr{M}_{3}$ for the set of numbers satisfying the conditions of Theorem 1 , we have the following estimate.

Theorem 2. The estimate

$$
\begin{aligned}
& \# \mathscr{M}_{3}(x) \geq \exp \left(\exp \left(\left(c_{1}+o(1)\right) \frac{\log \log x}{\log \log \log x}\right)\right) \\
& \text { holds as } x \rightarrow \infty,
\end{aligned}
$$

where $c_{1}=\log 2$.

Theorem 2 implies that the counting function of local minima $N \leq x$ exceeds $(\log x)^{A}$ for any positive constant $A$ (compare with (5)). In particular, the series

$$
\sum_{\substack{N>1 \text { local } \\ \text { minimum of } z(n)}} \frac{1}{(\log N)^{A}}
$$

diverges for all $A>0$.

\section{Proof of Theorem 1}

Suppose that $n=15$. Then $F_{n}=610$ and the only divisors $a$ of 610 satisfying (i) of Theorem 1 is $a \in\{1,2\}$. Now one checks that $z(609), z(611), z(304), z(306)$ are all larger than $z(305)=z(610)=15$. One does not even have to compute the above orders of appearance; one only has to factor the first 15 members of the Fibonacci sequence in order to convince oneself that none of them is a multiple of 609 or of 611 or of 304 or of 306 . From now on, $n \geq 16$.

Assume that $N=F_{n} / a$ satisfies the conditions of Theorem 1. Then $z(N)=n$. Indeed, $N \mid F_{n}$, so $z(N) \leq n$. On the other hand, if $N \mid F_{m}$ for some positive integer $m$, then (ii) of Theorem 1 shows that $p|N| F_{m}$ for some prime $p$ with $z(p)=n$; therefore $m \geq n$. Thus, $z(N)=n$. Assume now that $z(N+\varepsilon) \leq n$ for some $\varepsilon \in\{ \pm 1\}$. We then get an equation of the form

$$
\frac{F_{n}}{a}+\varepsilon=\frac{F_{m}}{b}
$$

for some positive integers $m \leq n$ and $b \mid F_{m}$. Since

$$
\frac{F_{n}}{a} \geq F_{n}^{4 / 5} \geq F_{16}^{4 / 5}>200,
$$

we get

$$
\frac{F_{m}}{b}=\frac{F_{n}}{a}+\varepsilon \geq \frac{F_{n}}{a}-1>\frac{2 F_{n}}{3 a},
$$

so

$$
b<\left(\frac{3 a}{2}\right)\left(\frac{F_{m}}{F_{n}}\right) \leq \frac{3 a}{2} .
$$

Let us see that in fact $m<n$. Indeed, if $m=n$, then multiplying both sides of (10) by $a b$, we get $F_{n}(a-b)=\varepsilon a b$. This implies first that $a \neq b$ and secondly that

$$
F_{n} \mid a b .
$$

But this conclusion is impossible because it leads, by (13), to

$$
F_{n} \leq a b<\frac{3 a^{2}}{2} \leq(1.5) F_{n}^{2 / 5}
$$

which is false for $n \geq 16$. Hence, $m \leq n-1$. Since

$$
\frac{F_{m}}{F_{n}} \leq \frac{F_{n-1}}{F_{n}} \leq \frac{2}{3}
$$

for $n \geq 16$, together with inequality (13), we get $b<a$. We will use the inequality

$$
\alpha^{k-2} \leq F_{k} \leq \alpha^{k-1}
$$

valid for all integers $k \geq 2$. We then have, using inequality (17) with $k=m$ and $n$, respectively, that

$$
\begin{aligned}
\alpha^{n-2} & \geq \alpha^{m-1} \geq \frac{F_{m}}{b} \geq \frac{F_{n}}{a}-1 \geq F_{n}^{4 / 5}-1>\frac{F_{n}^{4 / 5}}{\alpha^{2 / 5}} \\
& \geq \alpha^{4 n / 5-2},
\end{aligned}
$$

so $m=n-k$ for some integer $k \in[1, n / 5+1)$. Using Binet formula (3) with $i=m$ and $i=n$, respectively, (10) is equivalent to

$$
b\left(\alpha^{n}-\beta^{n}\right)+\varepsilon \sqrt{5} a b=a\left(\alpha^{n-k}-\beta^{n-k}\right),
$$

which can be regrouped as

$$
\alpha^{n}\left(b-a \alpha^{-k}\right)=b \beta^{n}-\varepsilon \sqrt{5} a b-a \beta^{n-k} .
$$

The number $\eta:=b-a \alpha^{-k}$ is an algebraic integer in $\mathbb{K}:=$ $\mathbb{Q}(\sqrt{5})$ which is not zero; otherwise $\alpha^{k}=b / a \in \mathbb{Q}$, which is 
impossible for positive integers $k$. Thus, the norm of $\eta$ over $\mathbb{K}$ is an integer which is at least 1 in absolute value. Hence,

$$
\left|b-a \alpha^{-k}\right|\left|b-a \beta^{-k}\right| \geq 1
$$

giving

$$
\begin{aligned}
\left|b-a \alpha^{-k}\right| & \geq \frac{1}{b+a \alpha^{k}}>\frac{1}{F_{n}^{1 / 5}\left(1+\alpha^{n / 5+1}\right)} \\
& >\frac{1}{\alpha^{(n-1) / 5}\left(1+\alpha^{(n+5) / 5}\right)} .
\end{aligned}
$$

Inserting (22) into (20) and using also (17), we get

$$
\begin{aligned}
\frac{\alpha^{(4 n+1) / 5}}{1+\alpha^{(n+5) / 5}} & <\alpha^{n}\left|b-a \alpha^{-k}\right|=\left|b \beta^{n}-\varepsilon \sqrt{5} a b-a \beta^{n-k}\right| \\
& \leq \frac{b}{\alpha^{n}}+\sqrt{5} a b+\frac{a}{\alpha^{n-k}} \\
& <\frac{F_{n}^{1 / 5}}{\alpha^{n}}+\sqrt{5} F_{n}^{2 / 5}+\frac{F_{n}^{1 / 5}}{\alpha^{4 n / 5-1}} \\
& <(\sqrt{5}+0.1) F_{n}^{2 / 5} \\
& <(\sqrt{5}+0.1) \alpha^{2(n-1) / 5}
\end{aligned}
$$

where we used the fact that $\alpha^{4 n / 5-1}>20$ for $n \geq 16$. The above inequality leads to the conclusion that $x:=\alpha^{n / 5}$ satisfies the inequality

$$
\alpha^{3 / 5} x^{2}-(\sqrt{5}+0.1) \alpha x-(\sqrt{5}+0.1)<0 .
$$

However, the largest root of the quadratic polynomial from the left-hand side above is $4.607 \cdots<4.664 \cdots=\alpha^{3.2} \leq$ $\alpha^{n / 5}=x$ for $n \geq 16$, so quadratic (24) in $x$ cannot be negative for $n \geq 16$, which is a contradiction.

The remaining assertions of the theorem are easy. To see unicity, assume that $N=F_{n} / a=F_{n^{\prime}} / a^{\prime}$ are two representations of the same $N$ satisfying conditions (i) and (ii) of the theorem. If $n=n^{\prime}$, then $a=a^{\prime}$ and we are through. If $n \neq n^{\prime}$, suppose without loss of generality that $n>n^{\prime}$. Then, by (ii), there is some primitive prime factor $p$ of $F_{n}$ which divides $N=F_{n} / a$. Since $p$ is primitive for $F_{n}$ it cannot divide $F_{n^{\prime}}$, which is a multiple of $N$, a contradiction. In particular, if $n \geq 15$ and $a \geq 2$, then $N=F_{n} / a$ cannot have another representation of the form $F_{n^{\prime}} / a^{\prime}$ with $a^{\prime}=1\left(\operatorname{so} n^{\prime}>n \geq 15\right)$, so it cannot be a Fibonacci number.

The theorem is therefore proved.

\section{Proof of Theorem 2}

Let $x$ be large and let $n$ be such that

$$
15 \leq n \leq c \log x
$$

Since $F_{n}<\alpha^{n} \leq x$ by (17), it follows that any number $N=F_{n} / a$ satisfying the conditions of Theorem 1 with $n$ satisfying (25) is in $\mathscr{M}_{3}(x)$. We now choose $n$ maximal satisfying inequality (25) of the form

$$
n=\prod_{i=1}^{m} p_{i}
$$

where $2=p_{1}<p_{2}<\cdots$ denotes the sequence of all primes. By the Prime Number Theorem, we have

$$
n=e^{(1+o(1)) p_{m}},
$$

as $m \rightarrow \infty$, showing that $p_{m}=\log \log x$ as $x \rightarrow \infty$. By the Prime Number Theorem again, we get that

$$
m=(1+o(1)) \frac{\log \log x}{\log \log \log x}
$$

as $x \rightarrow \infty$. Now let $a$ be a divisor of $F_{n / p_{m}}$. For large $x$, we have $p_{m} \geq 5$; therefore $a \leq F_{n / p_{m}} \leq F_{n}^{1 / p_{m}} \leq F_{n}^{1 / 5}$, so condition (i) from Theorem 1 is satisfied. Condition (ii) is also satisfied and in fact any primitive prime factor of $F_{n}$ will divide $F_{n} / F_{n / p_{m}}$, which is a divisor of $F_{n} / a$. Now by the Primitive Divisor Theorem, for every divisor $d \notin\{1,2,6\}$ of $n / p_{m}, F_{d}$ has a primitive prime factor $p_{d}$ which of course divides $F_{n / p_{m}}$. This shows that $F_{n / p_{m}}$ has at least

$$
\tau\left(\frac{n}{p_{m}}\right)-3=\frac{\tau(n)}{2}-3=2^{m-1}-3
$$

distinct prime factors, where $\tau(k)$ denotes the number of divisors of the positive integer $k$. Hence, the number of such convenient $a$ 's is at least as large as the number of square-free integers built up with prime factors from a set of $2^{m-1}-3$ distinct primes, and this number is at least as large as

$$
2^{2^{m-1}-3} \text {. }
$$

Thus,

$$
\# \mathscr{M}_{3}(x) \geq 2^{2^{m-1}-3},
$$

where $m$ satisfies estimate (28), which leads to the desired conclusion of the theorem.

\section{Conflict of Interests}

The authors declare that there is no conflict of interests regarding the publication of this paper.

\section{References}

[1] D. Marques, "On integer numbers with locally smallest order of appearance in the Fibonacci sequence," International Journal of Mathematics and Mathematical Sciences, vol. 2011, Article ID 407643, 4 pages, 2011.

[2] F. Luca and C. Pomerance, "On the local behavior of the order of appearance in the Fibonacci sequence," International Journal of Number Theory, vol. 10, no. 4, pp. 915-933, 2014.

[3] R. D. Carmichael, "On the numerical factors of the arithmetic forms $\alpha^{n} \pm \beta^{n}$," Annals of Mathematics, vol. 15, no. 2, pp. 30-70, 1913.

[4] Y. F. Bilu, G. Hanrot, and P. M. Voutier, "Existence of primitive divisors of Lucas and Lehmer numbers," Journal für die Reine und Angewandte Mathematik, vol. 539, pp. 75-122, 2001. 


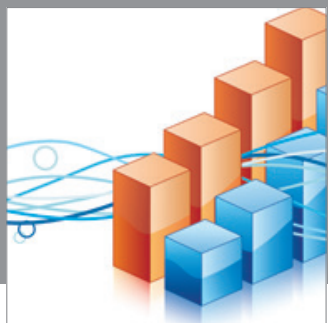

Advances in

Operations Research

mansans

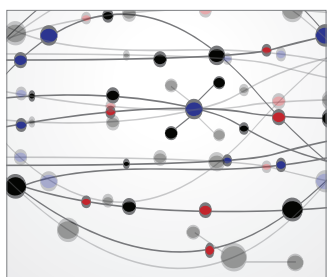

The Scientific World Journal
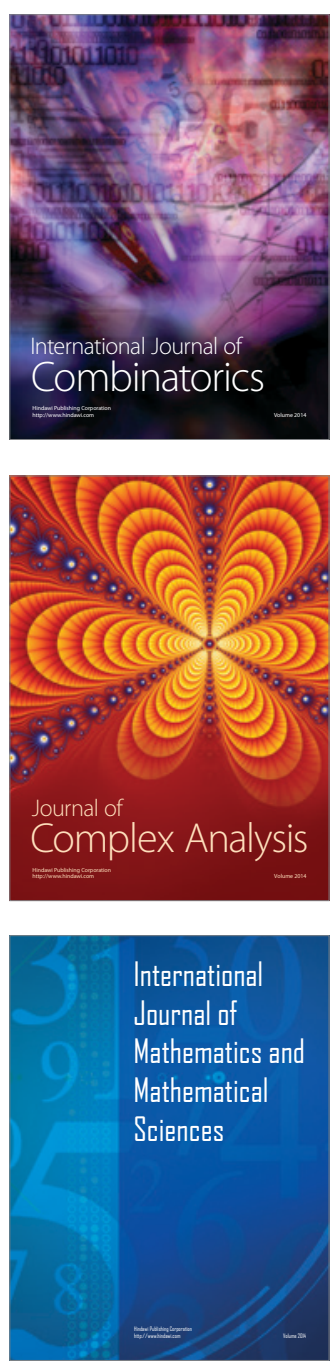
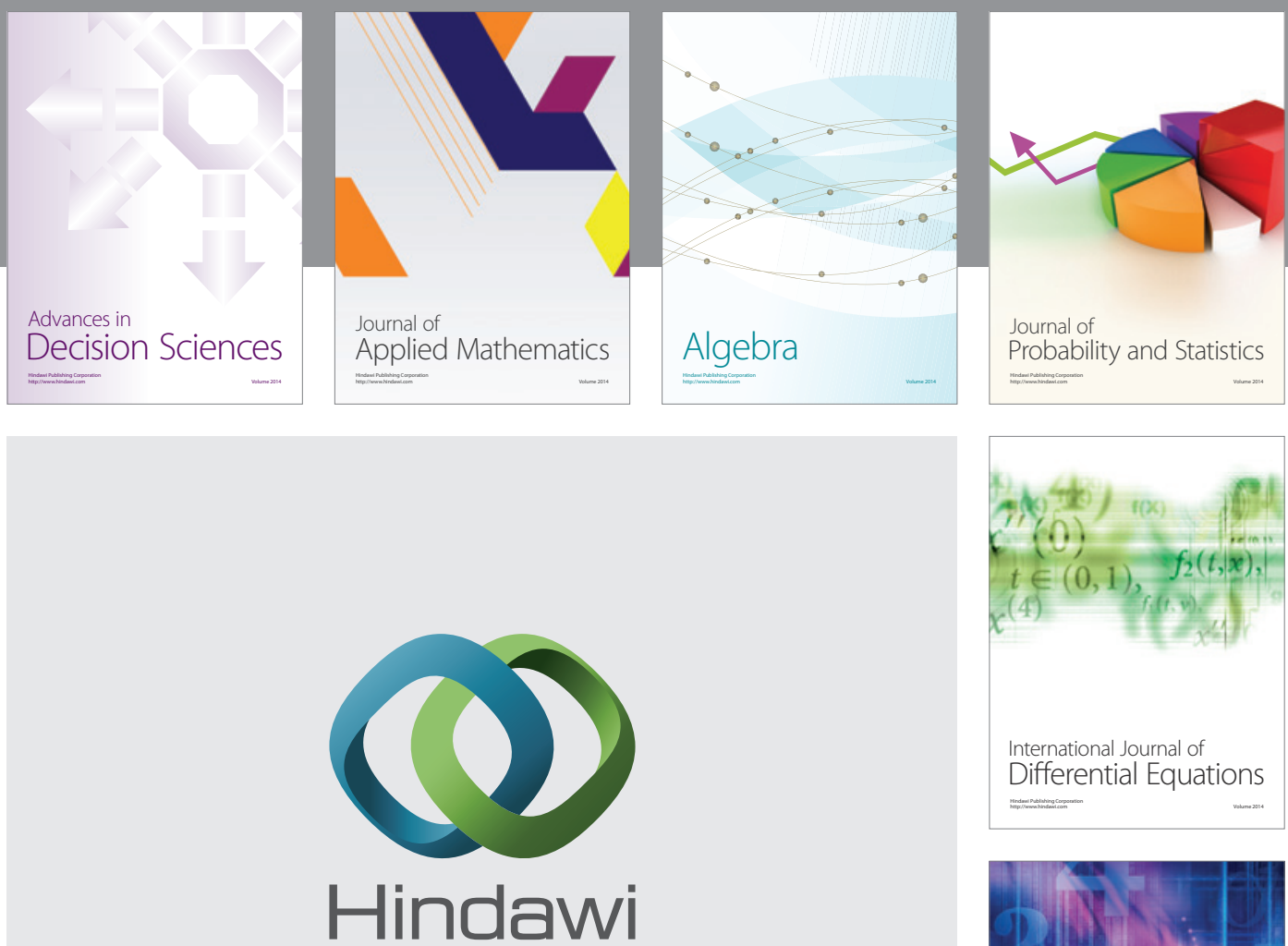

Submit your manuscripts at http://www.hindawi.com
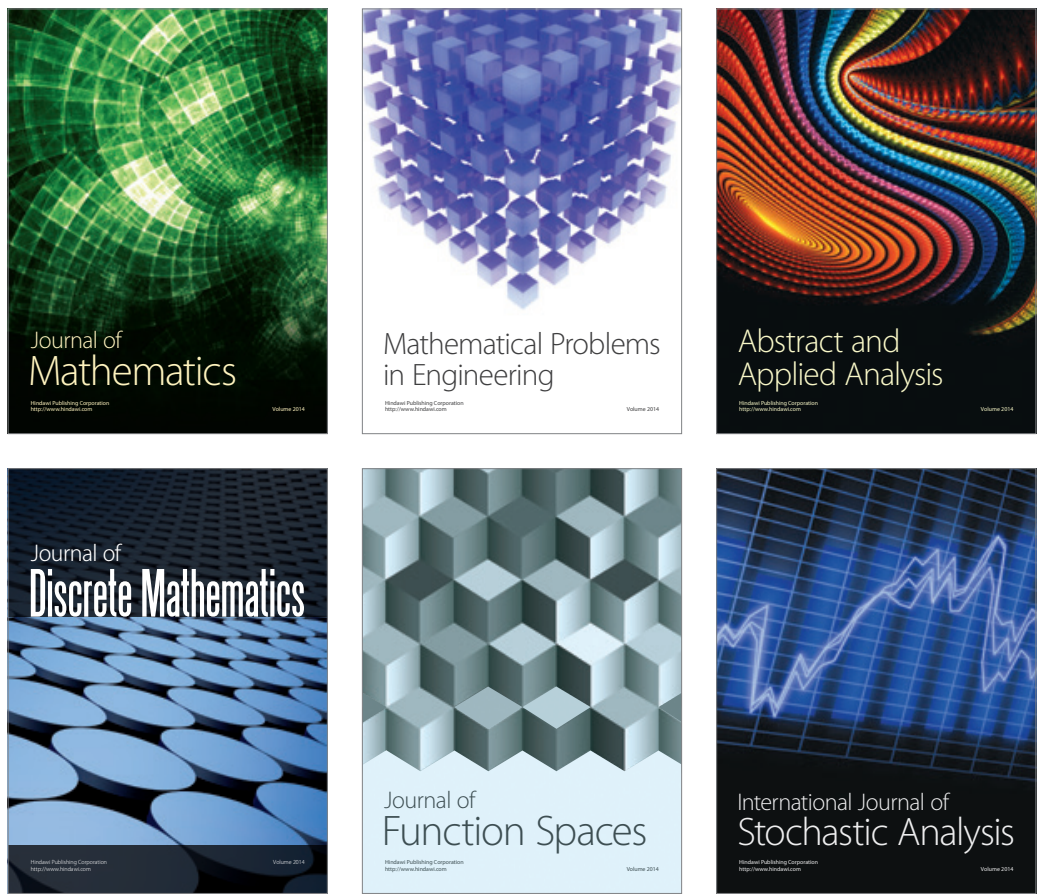

Journal of

Function Spaces

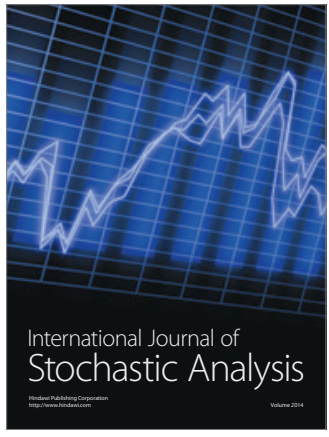

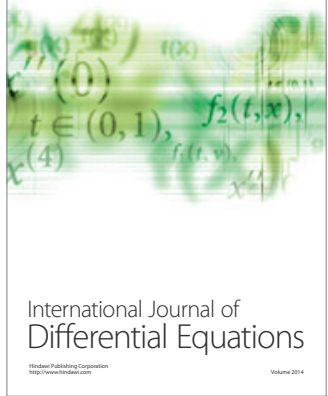
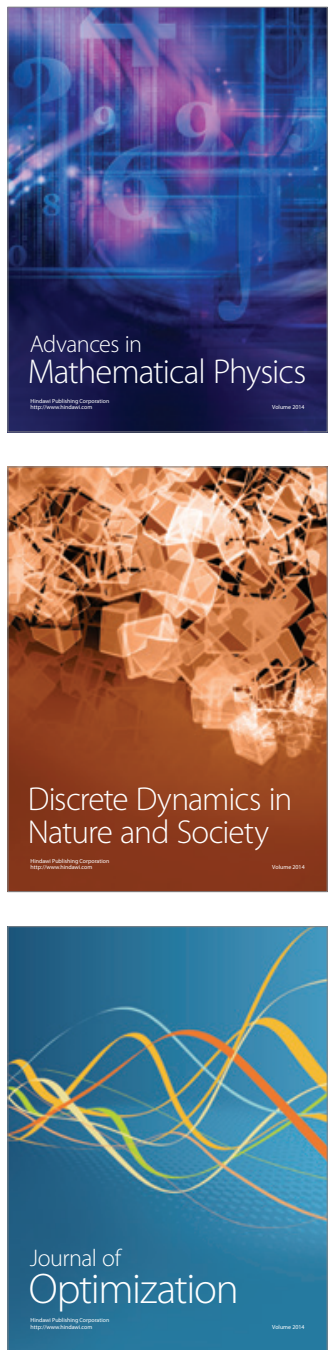